Supplementary Information

for

\title{
Glycine Residue Twists HOMO -.-HOMO Interactions in a Molecular Conductor
}

Abdelkrim El-Ghayoury*, Cécile Mézière, ${ }^{a}$ Sergey Simonov, ${ }^{b}$ Leokadiya Zorina, ${ }^{b}$ Pascale AubanSenzier, ${ }^{c}$ Pere Alemany, ${ }^{d}$ Enric Canadell, ${ }^{* e}$ and Patrick Batail ${ }^{*}, a$

a Laboratoire MOLTECH-Anjou, UMR 6200, CNRS, Université d'Angers, France

${ }^{b}$ Institute of Solid State Physics, Russian Academy of Sciences, Chernogolovka MD, Russia

c Laboratoire de Physique des Solides, Université Paris-Saclay, 91405 Orsay, France

${ }^{d}$ Departament de Ciència de Materials i Química Física and Institut de Química Teòrica i

Computacional (IQTCUB), Universitat de Barcelona, Martí i Franques 1, Barcelona 08028, Spain

e Institut de Ciència de Materials de Barcelona (ICMAB-CSIC), Campus de la UAB, 08193 Bellaterra, Spain

\section{Content}

Figure S1. DFT band structures for the metallic (a) and ferromagnetic (b) states of $\kappa$ $\left.(\mathbf{1})_{2}+\mathrm{HSO}_{4}^{-}\right]$using the single crystallographic cell.

Figure S2. DFT band structures calculated for the antiferromagnetic AF-2 and AF-3 states of $\kappa$ $(\mathbf{1})_{2}+\left[\mathrm{HSO}_{4}^{-}\right]$calculated using a double cell $\left(a^{\prime}=2 a, b^{\prime}=b\right.$ and $\left.c^{\prime}=c\right)$.

Figure S3. Extended Hückel band structure for the layers in $(\mathbf{1})_{2}{ }^{2+}\left[\mathrm{HO}_{2} \mathrm{C}-\mathrm{C}_{6} \mathrm{H}_{4}-\mathrm{SO}_{3}{ }^{-}\right]_{2}$. 

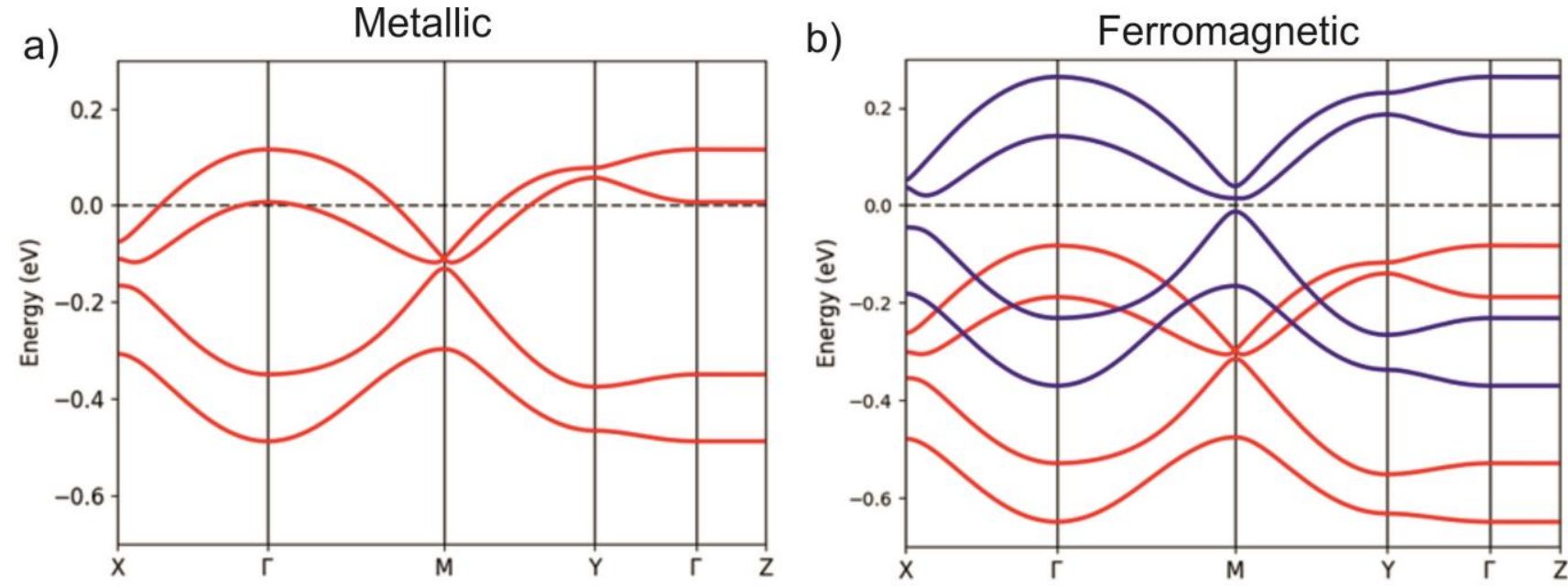

Figure S1. DFT band structures for the metallic (a) and ferromagnetic (b) states of $\kappa$ $(1)_{2}{ }^{+}\left[\mathrm{HSO}_{4}-\right]$ using the single crystallographic cell. $\Gamma=(0,0,0), \mathrm{Y}=\left(0, b^{*} / 2,0\right), \mathrm{Z}=\left(0,0, c^{*} / 2\right), \mathrm{M}$ $=\left(0, b^{*} / 2, c^{*} / 2\right)$ and $\mathrm{X}\left(a^{*} / 2,0,0\right)$. The dashed line refers to the highest occupied level in all cases. All levels are doubly filled in a). Spin-up and spin-down bands are shown in red and blue, respectively in b).

a)

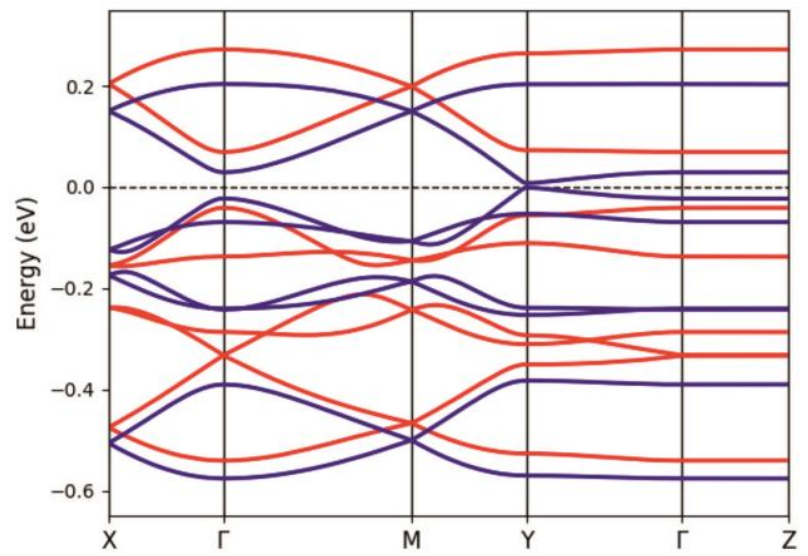

b)

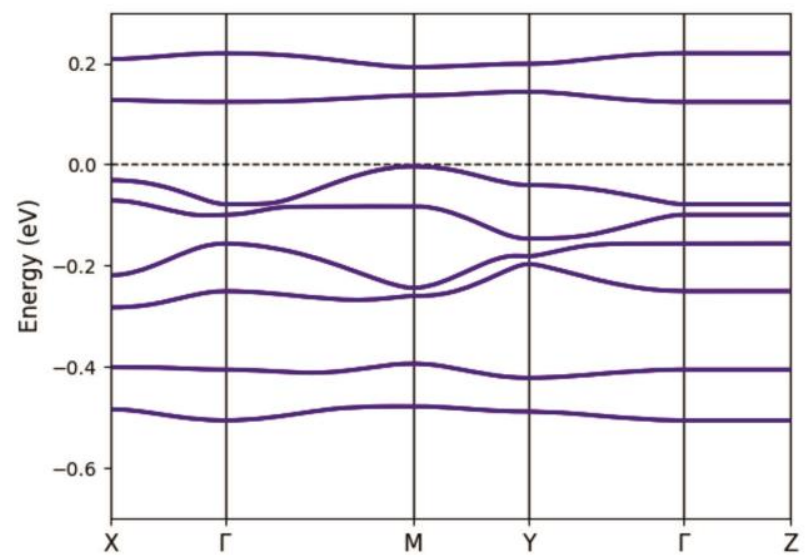

Figure S2. DFT band structures calculated for the antiferromagnetic AF-2 and AF-3 states of $\kappa$ $(1)_{2}{ }^{+}\left[\mathrm{HSO}_{4}^{-}\right]$calculated using a double cell $\left(a^{\prime}=2 a, b^{\prime}=b\right.$ and $\left.c^{\prime}=c\right) . \Gamma=(0,0,0), \mathrm{Y}=\left(0, b^{*} / 2,0\right), \mathrm{Z}$ $=\left(0,0, c^{*} / 2\right), \mathrm{M}=\left(0, b^{*} / 2, c^{*} / 2\right)$ and $\mathrm{X}\left(a^{*} / 2,0,0\right)$. The dashed line refers to the highest occupied level in all cases. All levels are doubly filled in a). Spin-up and spin-down bands are shown in red and blue, respectively. In b) the spin-up and spin-down bands are identical although located in spatially different but equivalent sites so that only the blue bands are visible. 


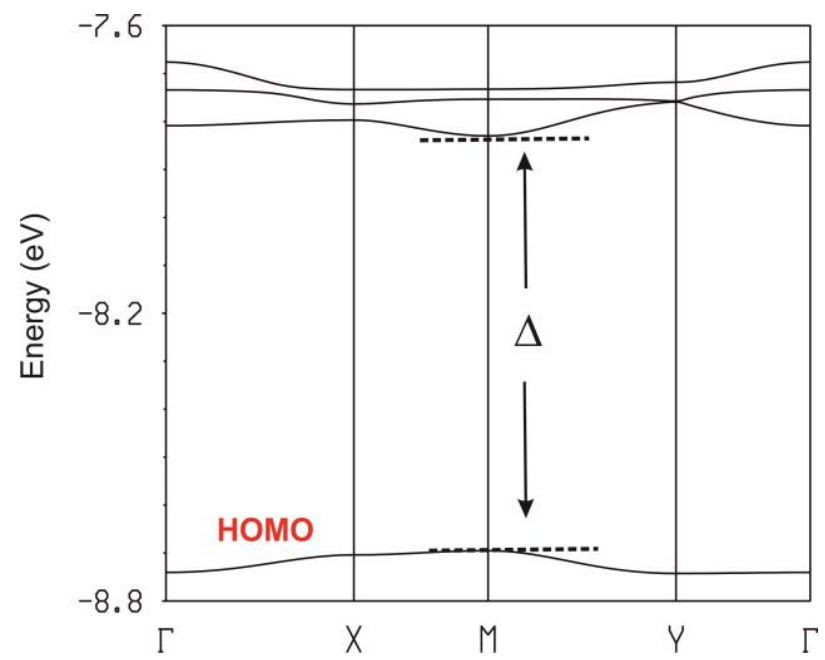

Figure S3. Extended Hückel band structure for the layers in $(\mathbf{1})_{2}{ }^{2+}\left[\mathrm{HO}_{2} \mathrm{C}-\mathrm{C}_{6} \mathrm{H}_{4}-\mathrm{SO}_{3}{ }^{-}\right]_{2}$. The donor HOMO band and the semiconductor band gap are highlighted. $\Gamma=(0,0), \mathrm{X}=\left(a^{*} / 2,0\right), \mathrm{Y}=$ $\left(0, b^{*} / 2\right)$ and $\mathrm{M}=\left(a^{*} / 2, b^{*} / 2\right)$. 